\title{
Recurrent paroxysmal supraventricular tachycardia in the beach chair position for shoulder surgery under general anesthesia
}

\author{
Kyung-Yoon Woo, Eun-Ju Kim, Ji-Hyang Lee, Sang Gon Lee, and Jong Seouk Ban \\ Department of Anesthesiology and Pain Medicine, Daegu Fatima Hospital, Daegu, Korea
}

The beach-chair position is commonly used for shoulder surgery. Its advantages include ease of setup, less intraoperative blood loss, and a lower incidence of traction neuropathy [1]. However, caution is required as the position may induce undesirable physiologic changes including decrease in mean arterial pressure and cardiac index, and increase in peripheral vascular resistance [2]. Other serious complications include venous air embolism [3] and hemodynamic instability [4]. We experienced a case of recurrent paroxysmal supraventricular tachycardia (PSVT) progressing to pulseless electrical activity accompanied by hypotension during shoulder surgery performed in the beach-chair position.

An 89-year-old female patient weighing $32 \mathrm{~kg}$ with a height of $145 \mathrm{~cm}$ presented for open reduction of nonunion of surgical neck fracture of the left humerus. She had been diagnosed with hypertension 5 years previously, and her current medications included benidipine, a calcium channel blocker, and thiazide, a diuretic. Preoperative laboratory tests were unremarkable. Chest radiography revealed cardiomegaly, and electrocardiogram showed left ventricular hypertrophy. Echocardiogram showed normal left ventricular systolic function with an ejection fraction of $65 \%$. No premedication was given. Vital signs checked upon arrival in the operating room revealed heart rate of 95 beats per minute, blood pressure of 160/90 $\mathrm{mmHg}$, and pulse oximetry at $95 \%$. For induction of anesthesia, $30 \mathrm{mg}$ of lidocaine, $50 \mathrm{mg}$ of propofol, and $30 \mathrm{mg}$ of rocuronium bromide were administered intravenously. Intubation was performed using a $7.0 \mathrm{~mm}$ cuffed tube. Anesthesia was maintained with $50 \%$
$\mathrm{N}_{2} \mathrm{O}-\mathrm{O}_{2}$, sevoflurane $1.5 \mathrm{vol} \%$, and remifentanil $0.05 \mu \mathrm{g} / \mathrm{kg} / \mathrm{min}$. For continuous monitoring of the arterial pressure and access to arterial blood gas analysis, a 22 gauge catheter was placed in the right radial artery. The patient's position was changed from supine to the beach-chair position. Invasive arterial blood pressure was measured with a transducer placed at the heart level. About 1-2 minutes after initiation of the beach-chair position, the blood pressure dropped to $85 / 35 \mathrm{mmHg}$. $50 \mu \mathrm{g}$ of phenylephrine was administered intravenously, and the operation was continued with dopamine being infused at $5 \mu \mathrm{g} / \mathrm{kg} / \mathrm{min}$. Blood pressure was maintained around 110/65 mmHg. About one hour after the operation had begun, sudden tachycardia with a heart rate of 140 beats per minute occurred for 3 seconds before returning to normal sinus rhythm. Ten minutes later, the tachycardia recurred with a heart rate of 140 beats per minute. Normal sinus rhythm was recovered after administration of 10 $\mathrm{mg}$ of intravenous esmolol. Five minutes after the second tachycardia, a heart rate of 150 beats per minute was noted. Again, normal sinus rhythm was recovered with $10 \mathrm{mg}$ of esmolol. However, as the basal heart rate was increased to 100 beats per minute, continuous administration of amiodarone at a rate of $15 \mathrm{mg}$ per minute was begun. In addition, under the impression of tachycardia caused by hypovolemia, transfusion of one pint of packed red cells was started. Tachycardia of 150 beats per minute occurred again five minutes after the third tachycardia. Blood pressure dropped to $60 / 40 \mathrm{mmHg}$ and echocardiogram showed loss of $\mathrm{p}$ waves and narrow QRS complexes (Fig. 1). The Valsalva maneuver was applied under the impression of PSVT

Corresponding author: Eun-Ju Kim, M.D., Department of Anesthesiology and Pain Medicine, Daegu Fatima Hospital, 576-31, Shinam-dong, Dong-gu, Daegu 701-600, Korea. Tel: 82-53-940-7434, Fax: 82-53-954-7417, E-mail: kej1127@fatima.or.kr

(c) This is an open-access article distributed under the terms of the Creative Commons Attribution Non-Commercial License (http:// creativecommons.org/licenses/by-nc/3.0/), which permits unrestricted non-commercial use, distribution, and reproduction in any medium, provided the original work is properly cited. 


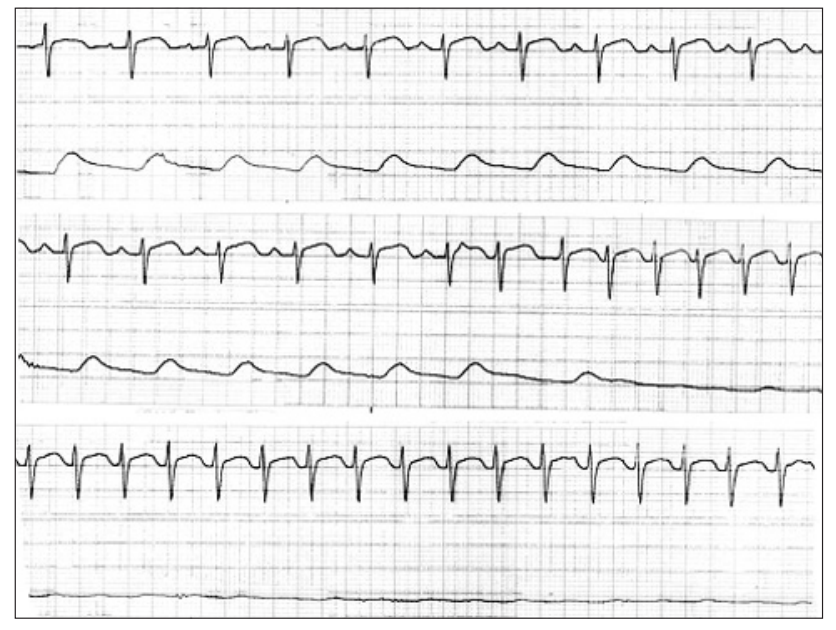

Fig. 1. Electrocardiogram and arterial pressure wave during the course of the operation. While electrocardiogram shifts from normal sinus rhythm to paroxysmal supraventricular tachycardia, arterial pressure gradually decreases to show a flat waveform.

with hypotension, but the technique was ineffective. Following immediate administration of $50 \mu \mathrm{g}$ of phenylephrine, normal sinus rhythm was recovered and blood pressure increased to 100/60 mmHg. Adenosine and a defibrillator were prepared for potential incidences of PSVT, and the surgeon was informed of the patient's condition. The operation was terminated 3 minutes later, and total blood loss was estimated at $300 \mathrm{ml}$. During the dressing of the operative site, tachycardia with a rate of 150 beats per minute occurred with hypotension of $40 / 30 \mathrm{mmHg}$. $50 \mu \mathrm{g}$ of phenylephrine was administered, but blood pressure failed to rise and normal sinus rhythm was not recovered. With adenosine prepared for administration, the patient was repositioned into the supine position. Normal sinus rhythm was recovered immediately after repositioning. Blood pressure rose to $100 / 60$ $\mathrm{mmHg}$, and heart rate was maintained at about 90 beats per minute. Following confirmation of hemodynamic stability, the patient was transferred to the postanesthesia care unit. Result from consultation to the Cardiology Department showed no specific findings on 24-hour Holter monitoring or cardiac markers. The patient was discharged two weeks after the operation without further problems.

PSVT accounts for roughly $2.5 \%$ of arrhythmias during anesthesia. Its prevalence is about twice as high in females as in males, and the risk is five times higher in patients above 65 years of age. Known causes of PSVT include underlying cardiogenic diseases, systemic diseases, thyrotoxicosis, digitalis intoxication, pulmonary embolism, and pregnancy. Exercise and stress can be triggering factors in young and healthy patients. Sympathomimetic drugs such as isoproterenol, parasympathetic inhibitors including atropine, and hyperventilation may also cause PSVT. When a patient is anesthetized, PSVT can be precipitated by changes in autonomic nervous system tone, by drug effects, or by intravascular volume shifts. Venous pooling, which is posture dependent, can rapidly reduce central blood volume, leading to decreases in cardiac filling pressure, stroke volume, and cardiac output. In standing position, $8-10 \mathrm{ml} / \mathrm{kg}$ of blood moves from the thorax to the lower extremities, a total volume of approximately 500-700 $\mathrm{ml}$ in normal healthy adults [5]. Since the patient is in the beach-chair position in the case described here, venous pooling and bleeding caused by the operation have reduced central blood volume. In addition, in elderly patients, reflex tachycardia caused by postural changes is reduced by vagal dysfunction, which makes them vulnerable to hypotension. Therefore, the vasoconstriction response is increased in order to compensate, which may increase sympathetic activity. It appears that, in the current case, many of the factors described earlier may have played roles in the occurrence of PSVT. However, the fact that blood pressure and sinus rhythm were recovered merely by postural change while anesthetic depth and drug concentration were consistent suggests the following: that blood pooling in the beach-chair position and decreased intravascular volume due to bleeding may have acted as the main factors in the occurrence of PSVT.

In conclusion, this case shows that the beach-chair position during general anesthesia may cause PSVT. In such a situation, hemodynamic instability and even pulseless electrical activity may occur depending on the patient's condition. Therefore, it appears that immediate repositioning to supine position is necessary for recovery of hemodynamic stability and normal sinus rhythm.

\section{References}

1. Skyhar MJ, Altchek DW, Warren RF, Wickiewicz TL, O'Brien SJ. Shoulder arthroscopy with the patient in the beach-chair position. Arthroscopy 1988; 4: 256-9.

2. Porter JM, Pidgeon C, Cunningham AJ. The sitting position in neurosurgery: a critical appraisal. Br J Anesth 1999; 82: 117-28.

3. Mammoto T, Hayashi Y, Ohnishi Y, Kuro M. Incidence of venous and paradoxical air embolism in neurosurgical patients in the sitting position: detection by transoesophageal echocardiography. Acta Anaesthesiol Scand 1998; 42: 643-7.

4. Kinsella SM, Tuckey JP. Perioperative bradycardia and asystole: relationship to vasovagal syncope and the Bezold-Jarisch reflex. Br J Anaesth 2001; 86: 859-68.

5. Smith JJ, Ebert TJ. General response to orthostatic stress. In: Circulatory Responses to the Upright Posture, Edited by Smith JJ. Boca Raton, FL, CRC Press. 1990, pp 1-46. 